\title{
RANDOM VECTOR VARIATIONAL INEQUALITIES AND RANDOM NONCOOPERATIVE VECTOR EQUILIBRIUM ${ }^{1}$
}

\author{
GUE MYUNG LEE \\ Pukyong National University, Department of Applied Mathematics \\ 599-1 Daeyeon-dong Nam-gu, Pusan 608-737, Korea \\ BYUNG SOO LEE \\ Kyungsung University, Department of Mathematics \\ 110-1 Daeyeon-dong, Nam-gu, Pusan 608-736, Korea \\ SHIH-SEN CHANG \\ Sichuan University, Department of Mathematics \\ Chengdu, Sichuan 610064, People's Republic of China
}

(Received September, 1995; Revised October, 1996)

\begin{abstract}
In this paper, we prove some existence theorems for random vector variational inequalities and an existence theorem for the random noncooperative vector equilibrium under suitable assumptions.

Key words: Random Vector Variational Inequalities, Random Noncooperative Vector Equilibrium, Random Vector Saddle Point Problem.
\end{abstract}

AMS subject classifications: $60 \mathrm{H} 25,90 \mathrm{~A} 14$.

\section{Introduction and Preliminaries}

Random variational inequalities and random equilibrium problems are of fundamental importance in modern random nonlinear analysis. To study the theory and applications of random variational inequalities and random equilibrium problems will not only exert a great influence in random nonlinear analysis but also provide forceful tools for various random equations, random control and abstract economics.

Recently, Tan [16] and Chang, et al. [3-5] considered random variational inequalities for real-valued functions, and gave some existence theorems of random solutions

\footnotetext{
${ }^{1}$ The first author was partially supported by Non-Directed Research Fund, Korea Research Foundation, 1995, and BSRI-96-1440. The second author was partially supported by BSRI-96-1405. The third author was partially supported by the National Natural Science Foundation of China.
} 
for their inequalities.

In this paper, we study random vector variational inequalities and random noncooperative vector equilibrium for vector-valued functions.

The paper is organized as follows. Section 2 deals with the existence problems of random solutions for some kinds of random vector variational inequalities which can be considered as random generalizations of the vector variational inequalities investigated by Chen and Yang [6]. Section 3 introduces the concept of random noncooperative vector equilibrium for vector-valued functions, which is a randomized and vector version of the ordinary noncooperative (scalar) equilibrium for real-valued functions. By using this concept and under suitable conditions, some existence theorems for the random noncooperative vector equilibrium are established. As its corollary, an existence theorem for a random vector saddle point problem is also proved.

For the sake of consistency, we will first give some definitions and preliminary results which will be needed in the upcoming sections.

Definition 1.1: Let $E$ be a vector space and $X$ be a convex subset of $E$. Let $Y$ be a topological vector space with a convex cone $K$ such that int $K \neq \emptyset$ and $K \neq Y$, and $g: X \rightarrow Y$ be a function, where int denotes interior. Then $g$ is said to be $K$-convex if for any $x, y \in X$ and $\lambda \in[0,1]$,

$$
g(\lambda x+(1-\lambda) y) \in \lambda g(x)+(1-\lambda) g(y)-K .
$$

Let $\boldsymbol{R}$ be the set of all real numbers and $\boldsymbol{R}^{+}=\{x \in \boldsymbol{R}: x \geq 0\}$.

Remark 1.1: When $Y=R$ and $K=R^{+}$, the $K$-convexity in Definition 1.1 reduces to the usual convexity.

Now we give the definition of Knaster-Kuratowski-Mazurkiewicz map (or KKM map, for short) and Fan-Knaster-Kuratowski-Mazurkiewicz theorem (or Fan-KKM theorem, for short) in [7].

Definition 1.2: Let $E$ be a vector space and $K$ be a nonempty subset of $E$. Then a set-valued map $G: K \rightarrow 2^{E}$ is called a KKM map if for each finite subset $\left\{x_{1}, \ldots, x_{n}\right\}$ of $K, \operatorname{co}\left\{x_{1}, \ldots, x_{n}\right\} \subset \bigcup_{i=1}^{n} G\left(x_{i}\right)$, where co denotes the convex hull.

Theorem 1.1: (Fan-KKM theorem). Let $E$ be a topological vector space, $K$ be a nonempty subset of $E$ and $G: K \rightarrow 2^{E}$ be a KKM map. If for any $x \in K, G(x)$ is closed in $E$ and there exists $x_{*} \in K$ such that $G\left(x_{*}\right)$ is compact, then $\bigcap_{x \in K} G(x) \neq \emptyset$.

Let $(\Omega, \mathcal{A})$ be a measurable space and $E$ be a topological space. We denote by $\mathfrak{B}(E)$ the $\sigma$-algebra of all Borel sets of $E$ and by $\mathcal{A} \times \mathfrak{B}(E)$ the collection of all the subsets of the form of $A \times B$, where $A \in \mathcal{A}$ and $B \in \mathscr{B}(E)$.

Definition 1.3: A set-valued map $F: \Omega \rightarrow 2^{E}$ is said to be $(\mathcal{A}, \mathscr{B}(E))$-measurable (or just measurable, for short) if for any $B \in \mathfrak{B}(E)$,

$$
F^{-1}(B):=\{\omega \in \Omega: F(\omega) \cap B \neq \emptyset\} \in \mathcal{A} \text {. }
$$

Definition 1.4: A Hausdorff topological space $E$ is called Suslin if there exist a Polish space (i.e., a separable complete metric space) $P$ and a continuous function $p$ from $P$ to $E$.

Lemma 1.1: [2]. Let $(\Omega, \mathcal{A})$ be a measurable space, $E$ be a separable metrizable space, $U$ be a metrizable space and $\varphi: \Omega \times E \rightarrow U$ be a function. If for any fixed $x \in E$, the function $\omega \mapsto \varphi(\omega, x)$ is measurable and for any fixed $\omega \in \Omega$, function $x \mapsto \varphi(\omega, x)$ is continuous, then $\varphi$ is measurable. 
Lemma 1.2: [2]. Let $E$ be a topological space and $X$ be a nonempty subset of $E$. Then $\mathfrak{B}(X)=\{B \cap X: B \in \mathscr{B}(E)\}$.

Theorem 1.2: $[14,15]$. Let $(\Omega, \mathcal{A})$ be a complete measurable space, $E$ be a Suslin space with the $\sigma$-algebra $\mathfrak{B}(E)$ of all Borel sets of $E$, and $F: \Omega \rightarrow 2^{E}$ be a set-valued map such that

$$
\operatorname{Graph}(F):=\{(\omega, x) \in \Omega \times X: x \in F(\omega)\} \in \mathcal{A} \times \mathfrak{B}(E) .
$$

Then there exists a measurable function $\xi: \Omega \rightarrow X$ such that $\xi(\omega) \in F(\omega)$ for all $\omega \in \Omega$.

Theorem 1.2 is known as the Aumann Theorem.

\section{Random Vector Variational Inequalities}

Now we give some existence theorems for random vector variational inequalities.

Theorem 2.1: Let $E$ be a Hausdorff topological vector space, $\mathfrak{B}(E)$ be the $\sigma$-algebra of all Borel sets of $E, X$ be a nonempty separable metrizable compact convex subset of $E,(\Omega, \mathcal{A})$ be a complete measurable space, $Y$ be a complete separable metrizable topological vector space with a convex cone $K$ such that int $K \neq \emptyset$ and $K \neq Y$, and $\mathfrak{B}(Y)$ be $\sigma$-algebra of all Borel sets of $Y$. Let $\varphi: \Omega \times X \times X \rightarrow Y$ be a vector-valued function.

If the conditions

(i) for any $x \in X, \varphi(\omega, x, \cdot)$ is $K$-convex and continuous;

(ii) for any $y \in X, \varphi(\omega, \cdot, y)$ is continuous;

(iii) for any $x, y \in X, \varphi(\cdot, x, y)$ is measurable;

(iv) $\varphi(\omega, x, x) \in K$ for any $x \in X$ and $\omega \in \Omega$, are satisfied, then there exists a measurable function $\xi: \Omega \rightarrow X$ such hat

$$
\varphi(\omega, \xi(\omega), y) \notin-\text { int } K \text { for all } y \in X \text { and } \omega \in \Omega \text {. }
$$

Proof: Define a set-valued map $G: \Omega \times X \rightarrow 2^{X}$ by

$$
G(\omega, y)=\{x \in X: \varphi(\omega, x, y) \notin-\operatorname{int} K\}, \quad(\omega, y) \in \Omega \times X
$$

and define a set-valued map for any fixed $y \in X, G_{y}: \Omega \rightarrow 2^{X}$ by

$$
G_{y}(\omega)=\{x \in X: \varphi(\omega, x, y) \notin-\operatorname{int} K\}, \omega \in \Omega .
$$

By Lemma 1.1 and Lemma 1.2 , for any fixed $y \in X, \varphi(\cdot, \cdot, y)$ is measurable and hence the graph of $G_{y}$ is as follows:

$$
\begin{gathered}
\operatorname{Graph}\left(G_{y}\right):=\left\{(\omega, y) \in \Omega \times X: x \in G_{y}(\omega)\right\} \\
=\{(\omega, x) \in \Omega \times X: \varphi(\omega, x, y) \in Y \backslash(-\operatorname{int} K)\} \in \mathcal{A} \times \mathfrak{B}(X) .
\end{gathered}
$$

Now we prove that for any fixed $\omega \in \Omega, G(\omega, \cdot): X \rightarrow 2^{X}$ is a KKM map. Indeed, suppose on the contrary that there exist a finite set $\left\{y_{1}, y_{2}, \ldots, y_{n}\right\} \in X$ and $z=$ $\sum_{i=1}^{n} a_{i} y_{i}\left(\sum_{i=1}^{n} a_{i}=1, a_{i} \geq 0\right)$ such that $z \notin \bigcup_{i=1}^{n} G\left(\omega, y_{i}\right)$. Then we have

$$
\varphi\left(\omega, z, y_{i}\right) \in-\operatorname{int} K, \quad i=1,2, \ldots, n
$$

and hence, by condition $(i)$,

$$
\varphi(\omega, z, z)=\varphi\left(\omega, z, \sum_{i=1}^{n} a_{i} y_{i}\right) \in \sum_{i=1}^{n} a_{i} \varphi\left(\omega, z, y_{i}\right)-K \subseteq-\operatorname{int} K-K=-\operatorname{int} K
$$


Since by condition $(i v), \varphi(\omega, z, z) \in K$, we have that $0 \in \operatorname{int} K$, which contradicts $K \neq Y$. Thus, for any fixed $\omega \in \Omega, G(\omega, \cdot): X \rightarrow 2^{X}$ is a KKM map. By condition (ii), for any fixed $\omega \in \Omega, G(\omega, y)$ is closed for any $y \in X$. Therefore, by Theorem 1.1,

$$
\bigcap_{y \in X} G(\omega, y) \neq \emptyset \text { for any fixed } \omega \in \Omega \text {. }
$$

Define a set-valued map $T: \Omega \rightarrow 2^{X}$ by

$$
T(\omega)=\bigcap_{y \in X} G(\omega, y), \omega \in \Omega .
$$

Then by $(2.2), T(\omega) \neq \emptyset$ for all $\omega \in \Omega$.

Since $X$ is separable, there exists a sequence $\left\{y_{i}\right\}_{i=1}^{\infty}$ in $X$ such that closure of $\left\{y_{i}\right\}_{i=1}^{\infty}$ equals $X$.

Now we prove that $\bigcap_{y \in X} G(\omega, y)=\bigcap_{i=1}^{\infty} G\left(\omega, y_{i}\right)$.

It is clear that $\bigcap_{y \in X} G(\omega, y) \subseteq \bigcap_{i=1}^{\infty} G\left(\omega, y_{i}\right)$. Suppose that $\bigcap_{i=1}^{\infty} G\left(\omega, y_{i}\right) \not \subset$ $\bigcap_{y \in X} G(\omega, y)$. Then there exists $x_{0} \in \bigcap_{i=1}^{\infty} G\left(\omega, y_{i}\right)$ but $x_{0} \notin \bigcap_{y \in X} G(\omega, y)$. Hence $x_{0} \in$ $\bigcap_{i=1}^{\infty} G\left(\omega, y_{i}\right)$ and there exists $y_{0} \in X$ such that $x_{0} \notin G\left(\omega, y_{0}\right)$, i.e.,

$$
\varphi\left(\omega, x_{0}, y_{0}\right) \in-\operatorname{int} K \text {. }
$$

Furthermore, there exists a subsequence $\left\{y_{n_{j}}\right\}_{j=1}^{\infty}$ of $\left\{y_{i}\right\}_{i=1}^{\infty}$ such that $y_{n_{j}} \rightarrow y_{0}$. Since $x_{0} \in \bigcap_{j=1}^{\infty} G\left(\omega, y_{n_{j}}\right), \varphi\left(w, x_{0}, y_{n_{j}}\right) \in Y \backslash(-\operatorname{int} K)$. By condition $(i), \varphi\left(w, x_{0}, y_{0}\right)$ $=\lim _{j \rightarrow \infty} \varphi\left(\begin{array}{c}j, x_{0}, y_{n_{j}} \\ )\end{array}\right) Y Y(-\operatorname{int} K)$ which contradicts (2.3). Hence, we have that $\bigcap_{y \in X}^{j \rightarrow \infty} G(\omega, y)=\bigcap_{i=1}^{\infty} G\left(\omega, y_{i}\right)$. Moreover, we have

$$
\begin{gathered}
\operatorname{Graph}(T):=\left\{(\omega, x) \in \Omega \times X: x \in T(\omega)=\bigcap_{i=1}^{\infty} G\left(\omega, y_{i}\right)\right\} \\
=\bigcap_{i=1}^{\infty}\left\{(\omega, x) \in \Omega \times X: x \in G_{y_{i}}(\omega)\right\} \\
=\bigcap_{i=1}^{\infty} \operatorname{Graph}\left(G_{y_{i}}\right) \in \mathcal{A} \times \mathfrak{B}(X) \quad(\text { by }(2.1)) .
\end{gathered}
$$

By Theorem 1.2, there exists a measurable function $\xi: \Omega \rightarrow X$ such that $\xi(\omega) \in$ $\bigcap G(\omega, y)$ for all $\omega \in \Omega$. Thus, $\varphi(\omega, \xi(\omega), y) \notin-\operatorname{int} K$ for all $y \in X$ and $\omega \in \Omega$.

$y \in X$ This completes the proof of Theorem 2.1.

The following lemma is a generalization of Lemma B in Kum [10]. This lemma has been established in [12], but to make the upcoming results self-contained we repeat the proof of this lemma again.

Lemma 2.1: Let $E, Y$ be two locally convex Hausdorff topological vector spaces and $X$ be a bounded subset of $E$. Let $L(E, Y)$ be the set of all continuous linear functions from $E$ to $Y$, equipped with the topology of bounded convergence. Define a vector-valued function $\phi: L(E, Y) \times X \rightarrow Y$ by $\phi(f, x)=f(x), f \in L(E, Y)$ and $x \in X$. Then $\phi$ is continuous.

Proof: Denote $f(x)=\langle f, x\rangle$, and let $\left(f_{\nu}, x_{\nu}\right)$ be a net convergent to $(f, x)$ in $L(E, Y) \times X$. then $f_{\nu} \rightarrow f$ and $x_{\nu} \rightarrow x$. Consider the following equality 


$$
\left\langle f_{\nu}, x_{\nu}\right\rangle-\langle f, x\rangle=\left\langle f_{\nu}-f, x_{\nu}\right\rangle+\left\langle f, x_{\nu}-x\right\rangle .
$$

Since $L(E, Y)$ is equipped with the topology of bounded convergence, from the above equality, we can easily verify that $\left\langle f_{\nu}, x_{\nu}\right\rangle \rightarrow\langle f, x\rangle$. Hence $\phi$ is continuous.

This completes the proof of Lemma 2.1.

Theorem 2.2: Let $E$ be a locally convex Hausdorff topological vector space, $\mathfrak{B}(E)$ be the $\sigma$-algebra of all Borel sets of $E, X$ be a nonempty separable metrizable compact convex subset of $E,(\Omega, \mathcal{A})$ be a complete measurable space, $Y$ be a complete separable metrizable topological vector space with a convex cone $K$ such that int $K \neq \emptyset$ and $K \neq Y$, and $\mathscr{B}(Y)$ be the $\sigma$-algebra of all Borel sets of $Y$. Let $L(E, Y)$ be the set of all continuous linear functions from $E$ to $Y$, equipped with the topology of bounded convergence and $f: \Omega \times X \rightarrow L(E, Y)$ be a vector-valued function satisfying the conditions

(i) for any $\omega \in \Omega, f(\omega, \cdot)$ is continuous; and

(ii) for any $x \in X, f(\cdot, x)$ is measurable.

Then there exists a measurable function $\xi: \Omega \rightarrow X$ such that

$$
\langle f(\omega, \xi(\omega)), y-\xi(\omega)\rangle \notin-\text { int } K \text { for all } y \in X \text { and } \omega \in \Omega .
$$

Proof: Let $\varphi(\omega, x, y)=\langle f(\omega, x), y-x\rangle$. Then by Lemma 2.1, we can easily see that $\varphi$ satisfies all conditions of Theorem 2.1. Therefore, by Theorem 2.1, there exists a measurable function $\xi: \Omega \rightarrow X$ such that

$$
\langle f(\omega, \xi(\omega)), y-\xi(\omega)\rangle \notin-\operatorname{int} K \text { for all } y \in X \text { and } \omega \in \Omega .
$$

This completes the proof of Theorem 2.2.

Remark 2.1: (1) Theorem 2.2 is the vector version of the existence theorem for random variational inequality, which was investigated by Chang et al. [5]. Therefore (2.3) in Theorem 2.2 is the randomized and vector version of Hartmann-Stampacchia variational inequality [9].

(2) Theorem 2.2 is the randomized version of the existence theorem for vector variational inequality studied by Chen and Yang [6].

\section{Random Noncooperative Vector Equilibrium}

Here we define the random noncooperative vector equilibrium for vector-valued functions.

Let $X:=\prod_{i=1}^{n} X^{i}$ be a nonempty subset of the product space $E:=\prod_{i=1}^{n} E^{i}$ where $E^{i}$ is a Hausdorff topological vector space, and $X^{i}$ is a nonempty subset of $E^{i}$. Let $\mathscr{B}\left(E^{i}\right)$ be the $\sigma$-algebra of all Borel sets of $E^{i},(\Omega, \mathcal{A})$ be a complete measurable space, $Y$ be a complete separable metrizable topological vector space with a convex cone $K$ such that int $K \neq Y$ and $K \neq Y$, and $\mathscr{B}(Y)$ be the $\sigma$-algebra of all Borel sets of $Y$. Let $G_{i}: \Omega \times X \rightarrow Y$ be a vector-valued function, and $x^{\hat{i}}=\left(x^{1}, \ldots, x^{i-1}, x^{i+1}, \ldots, x^{n}\right) \in$ $\prod_{j \neq i} X^{j}$ and $x\left(x^{\hat{i}}, x^{i}\right) \in \prod_{j \neq i} X^{j} \times X^{i}, i=1, \ldots, n$, for any $x=\left(x^{1}, \ldots, x^{n}\right) \in X$.

Definition 3.1: Let $\xi(\omega)=\left(\xi^{1}(\omega), \ldots, \xi^{n}(\omega)\right): \Omega \rightarrow X$ be a measurable function. We say that $\xi$ is a random noncooperative vector equilibrium if for each $i \in$ $\{1,2, \ldots, n\}$, we have 


$$
G_{i}\left(\omega, \xi^{\hat{i}}(\omega), y^{i}\right)-G_{i}\left(\omega, \xi^{\hat{i}}(\omega), \xi^{i}(\omega)\right) \notin-\operatorname{int} K \text { for any } y^{i} \in X^{i} \text { and } \omega \in \Omega \text {. }
$$

Remark 3.1: Definition 3.1 is a randomized and vector version of the ordinary noncooperative (scalar) equilibrium in $[1,8,13]$.

Now we prove the existence theorem for the random noncooperative vector equilibrium in the sense of Definition 3.1.

Theorem 3.1: Suppose that the following conditions are satisfied:

(i) for each $i \in\{1, \ldots, n\}, X^{i}$ is a nonempty, separable, metrizable, compact and convex subset of $E^{i}$;

(ii) for any fixed $x^{i} \in \prod_{j \neq i} X^{j}$ and $\omega \in \Omega$, the function $y^{i} \mapsto G_{i}\left(\omega, x^{\widehat{i}}, y^{i}\right)$ is $K$ convex;

(iii) for any fixed $\omega \in \Omega, G_{i}(\omega, \cdot)$ is continuous;

(iv) for any fixed $x \in X, G_{i}(\cdot, x)$ is measurable.

Then there exists a random noncooperative vector equilibrium.

Proof: Define a vector-valued function $G: \Omega \times X \times X \rightarrow Y$ by

$$
G(\omega, x, y)=\sum_{i=1}^{n}\left[G_{i}\left(\omega, x^{\hat{i}}, y_{i}\right)-G_{i}\left(\omega, \widehat{i}, x^{i}\right)\right], \omega \in \Omega \text { and } x, y \in X .
$$

Then for all $x \in X, G(\omega, x, x)=0 \in K$. By the condition $(i), X$ is a nonempty separable metrizable compact convex subset of a Hausdorff topological vector space $E$. By condition (ii), the function $y \mapsto G(\omega, x, y)$ is $K$-convex. By condition(iii), for any fixed $\omega \in \Omega$, the function $x \mapsto G(\omega, x, y)$ is continuous. By condition $(i v)$, for any $x, y \in X, G(\cdot, x, y)$ is measurable.

By Theorem 2.1, there exists a measurable function $\xi: \Omega \rightarrow X$ such that

$$
G(\omega, \xi(\omega), y) \notin-\operatorname{int} K \text { for all } y \in X \text { and } \omega \in \Omega \text {. }
$$

Let $\xi(\omega)=\left(\xi^{1}(\omega), \ldots, \xi^{n}(\omega)\right), \omega \in \Omega$. Then $\xi^{i}: \Omega \rightarrow X_{\curlywedge}^{i}$ is a measurable function. For each $i \in\{1, \ldots, n\}$ and any $y^{i} \in X^{i}$, let us take $y=\left(\xi^{i}(\omega), y^{i}\right)$. Then from (3.1),

$$
G(\omega, \xi(\omega), y)=\sum_{i=1}^{n}\left[G_{i}\left(\omega, \xi^{\hat{i}}(\omega), y^{i}\right)-G_{i}\left(\omega, \xi^{i}(\omega), \xi^{i}(\omega)\right)\right] \notin-\operatorname{int} K .
$$

Therefore, for each $i \in\{1, \ldots, n\}$, we have

$$
G_{i}\left(\omega, \xi^{\hat{i}}(\omega), y^{i}\right)-G_{i}\left(\omega, \xi^{\hat{i}}(\omega), \xi^{i}(\omega)\right) \notin-\operatorname{int} K
$$

for any $y^{i} \in X^{i}$ and $\omega \in \Omega$; that is, $\xi$ is a random noncooperative vector equilibrium.

This completes the proof of Theorem 3.1.

Remark 3.2: (1) The above Theorem 3.1 is a randomized and vector version of the existence theorem for an ordinary noncooperative (scalar) equilibrium in $[1,8$, 13].

(2) The above Theorem 3.1 can be regarded as a randomized version of the existence theorem for a noncooperative vector equilibrium in Lee et al. [11].

Let $E^{1}$ and $E^{2}$ be two Hausdorff topological vector spaces, $\mathfrak{B}\left(E^{i}\right), i=1,2$, be the $\sigma$-algebra of all Borel sets of $E^{i}, i=1,2,(\Omega, \mathcal{A})$ be a complete measurable space, $Y$ be a complete separable metrizable topological vector space with a convex cone $K$ such that int $K \neq Y$ and $K \neq Y$, and $\mathscr{B}(Y)$ be the $\sigma$-algebra of all Borel sets of $Y$. Let $X^{i} \subset E^{i}, i=1,2$ and $F: \Omega \times X^{1} \times X^{2} \rightarrow Y$ be a vector-valued function.

From Theorem 3.1 , we can obtain the following random vector saddle point theorem.

Theorem 3.2: Suppose that the following conditions are satisfied; 
(i) $X^{1}$ and $X^{2}$ are nonempty, separable, metrizable, compact and convex subsets of $E^{1}$ and $E^{2}$, respectively;

(ii) for any fixed $x^{1} \in X^{1}$ and $\omega \in \Omega$, the function $x^{2} \mapsto F\left(\omega, x^{1}, x^{2}\right)$ is $K$-concave, and for any fixed $x^{2} \in X^{2}$ and $\omega \in \Omega$, the function $x^{1} \mapsto F\left(\omega, x^{1}, x^{2}\right)$ is $K$-convex;

(iii) for any fixed $\omega \in \Omega, F(\omega, \cdot, \cdot)$ is continuous;

(iv) for any fixed $\left(x^{1}, x^{2}\right) \in X^{1} \times X^{2}, F\left(\cdot, x^{1}, x^{2}\right)$ is measurable.

Then there exist measurable functions $\xi^{i}: \Omega \rightarrow X, i=1,2$, satisfying the following random vector saddle point problem:

Find measurable functions $\xi^{i}: \Omega \rightarrow X^{i}, i=1,2$ such that

$$
\begin{gathered}
F\left(\omega, \xi^{1}(\omega), x^{2}\right)-F\left(\omega, \xi^{1}(\omega), \xi^{2}(\omega)\right) \notin-i n t K \text { and } \\
F\left(\omega, \xi^{1}(\omega), \xi^{2}(\omega)\right)-F\left(\omega, x^{1}, \xi^{2}(\omega)\right) \notin \text { int } K,
\end{gathered}
$$

for any $x^{1} \in X^{1}, x^{2} \in X^{2}$ and $\omega \in \Omega$.

Proof: Let $G_{1}\left(\omega, x^{1}, x^{2}\right)=F\left(\omega, x^{1}, x^{2}\right), G_{2}\left(\omega, x^{1}, x^{2}\right)=-F\left(\omega, x^{1}, x^{2}\right)$ and $n=2$. Then all the assumptions of Theorem 3.1 are satisfied. Hence, by Theorem 3.1 , there exist measurable functions $\xi^{i}: \Omega \rightarrow X^{i}, i=1,2$ such that

$$
\begin{gathered}
G_{1}\left(\omega, \xi^{1}(\omega), x^{2}\right)-G_{1}\left(\omega, \xi^{1}(\omega), \xi^{2}(\omega)\right) \notin-\operatorname{int} K \text { and } \\
G_{2}\left(\omega, x^{1}, \xi^{2}(\omega)\right)-G_{2}\left(\omega, \xi^{1}(\omega), \xi^{2}(\omega)\right) \notin-\operatorname{int} K
\end{gathered}
$$

for any $x^{1} \in X^{2}, x^{2} \in X^{2}$, and $\omega \in \Omega$.

Hence we have

$$
\begin{gathered}
F\left(\omega, \xi^{1}(\omega), x^{2}\right)-F\left(\omega, \xi^{1}(\omega), \xi^{2}(\omega)\right) \notin-\operatorname{int} K \text { and } \\
F\left(\omega, \xi^{1}(\omega), \xi^{2}(\omega)\right)-F\left(\omega, x^{1}, \xi^{2}(\omega)\right) \notin \operatorname{int} K
\end{gathered}
$$

for any $x^{1} \in X^{1}, x^{2} \in X^{2}$, and $\omega \in \Omega$.

This completes the proof of Theorem 3.2.

For $Y=\boldsymbol{R}$ and $K=\boldsymbol{R}^{+}$, Theorem 3.2 yields the following corollary.

Corollary 3.1: Let $X^{i} \subset E^{i}, i=1,2$ and $f: \Omega \times X^{1} \times X^{2} \rightarrow \boldsymbol{R}$ be a real-valued function.

If the following conditions are satisfied:

(i) $\quad X^{1}$ and $X^{2}$ are nonempty, separable, metrizable, compact and convex subsets of $E^{1}$ and $E^{2}$, respectively;

(ii) for any fixed $x^{1} \in X^{1}$ and $\omega \in \Omega$, the function $x^{2} \mapsto f\left(\omega, x^{1}, x^{2}\right)$ is concave (in the usual sense), and for any fixed $x^{2} \in X^{2}$ and $\omega \in \Omega$, the function $x^{1} \mapsto f\left(\omega, x^{1}, x^{2}\right)$ is convex (in the usual sense);

(iii) for any fixed $\omega \in \Omega, f(\omega, \cdot, \cdot)$ is continuous;

(iv) for any fixed $\left(x^{1}, x^{2}\right) \in X^{1} \times X^{2}, f\left(\cdot, x^{1}, x^{2}\right)$ is measurable,

then there exist measurable functions $\xi^{i}: \Omega \rightarrow X^{i}, i=1,2$, satisfying the following random saddle point problem:

Find measurable functions $\xi^{i}: \Omega \rightarrow X^{i}, i=1,2$ such that

$$
f\left(\omega, \xi^{1}(\omega), x^{2}\right) \leq f\left(\omega, \xi^{1}(\omega), \xi^{2}(\omega)\right) \leq f\left(\omega, x^{1}, \xi^{2}(\omega)\right)
$$

for any $x^{1} \in X^{1}, x^{2} \in X^{2}$, and $\omega \in \Omega$.

Remark 3.3: Chang et al. [5] considered a random saddle point problem for realvalued functions, and proved an existence theorem of random solutions for random saddle point problem under the quasiconvexity and quasiconcavity assumptions, and 
some additional ones.

\section{References}

[1] Aubin, J.P., Applied Abstract Analysis, John Wiley, New York, London 1977.

[2] Castaing, C. and Valadier, M., Convex Analysis and Measurable Multifunctions, Lecture Notes in Mathematics 580, Springer-Verlag, Berlin, New York 1977.

[3] Chang, S.S., Variational Inequality and Complementarity Problem Theory with Applications, Shanghai Scientific and Technological Literature Publishing House, Shanghai 1991 (in Chinese).

[4] Chang, S.S. and Zhu, Y.G., On the problems for a class of random variational inequalities and random quasi-variational inequalities, J. Math. Res. Expos. 9:3 (1989), 385-393 (in Chinese).

[5] Chang, S.S. and Qing, L., Random variational inequalities and random saddle point theorems, Applied Math. and Mech. (Special issue) (1993), 132-137 (in Chinese).

[6] Chen, G.Y. and Yang, X.Q., The vector complementarity problem and its equivalence with the weak minimal element in ordered sets, J. Math. Anal. Appl. 153 (1990), 136-158.

[7] Fan, K., A generalization of Tychonoff's fixed point theorem, Math. Ann. 142 (1961), 305-310.

[8] Fan, K., Applications of a theorem concerning sets with convex sections, Math. Ann. 163 (1966), 189-203.

[9] Hartmann, P. and Stampacchia, G., On some nonlinear elliptic differential functional equations, Acta Math. 115 (1966), 271-310.

[10] Kum, S., A generalization of generalized quasi-variational inequalities, J. Math. Anal. Appl. 182 (1994), 158-164.

[11] Lee, G.M., Kim, D.S. and Lee, B.S., On noncooperative vector equilibrium, Indian J. Pure Appl. Math 27 (1996), 735-739.

[12] Lee, G.M., Lee, B.S. and Chang, S.S., On vector quasivariational inequalities, J. Math. Anal. Appl. (to appear).

[13] Nash, J., Non-cooperative games, Ann. of Math. 54 (1951), 286-295.

[14] Papageorgiou, N.S., Random fixed point theorems for measurable multifunctions in Banach space, Proc. Amer. Math. Soc. 97 (1986), 507-514.

[15] Saint-Beuve, M., On the existence of Von Neumann-Aumann's theorem, $J$. Funct. Anal. 17 (1974), 112-129.

[16] Tan, N.X., Random variational inequalities, Math. Nachr. 125 (1986), 319-328. 


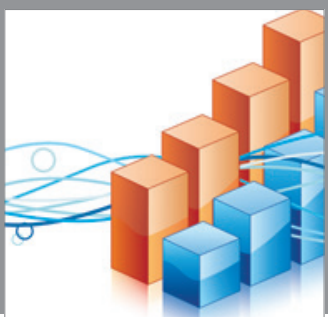

Advances in

Operations Research

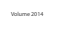

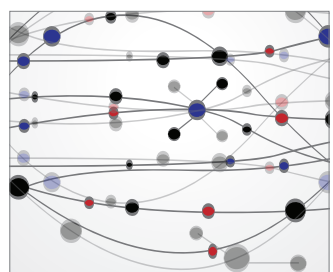

\section{The Scientific} World Journal
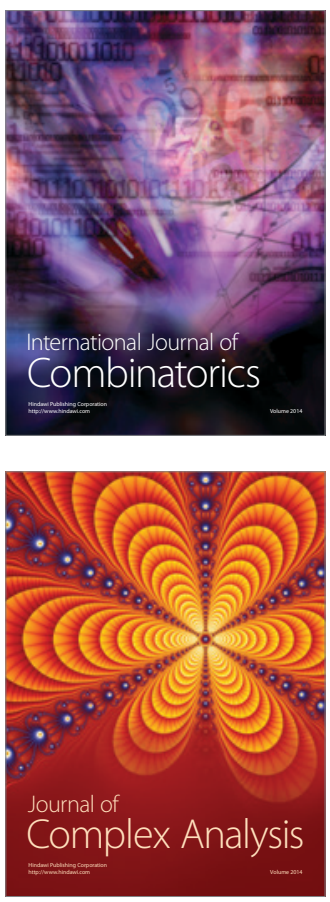

International Journal of

Mathematics and

Mathematical

Sciences
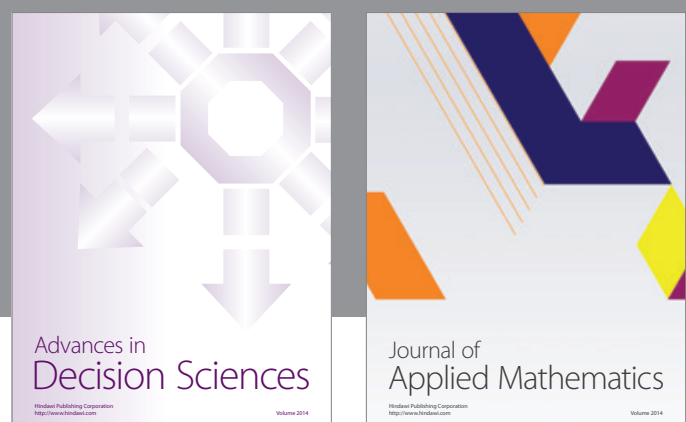

Journal of

Applied Mathematics
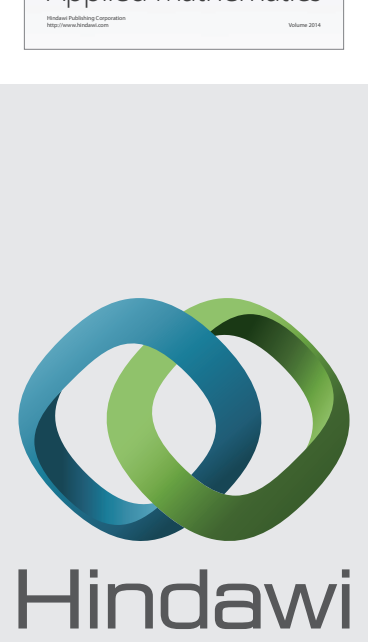

Submit your manuscripts at http://www.hindawi.com
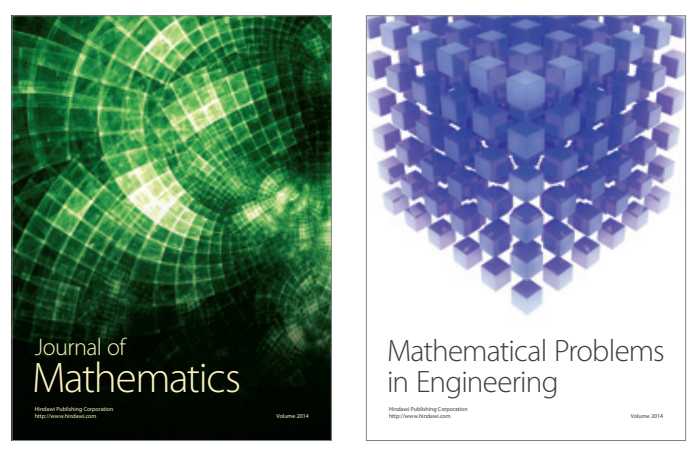

Mathematical Problems in Engineering
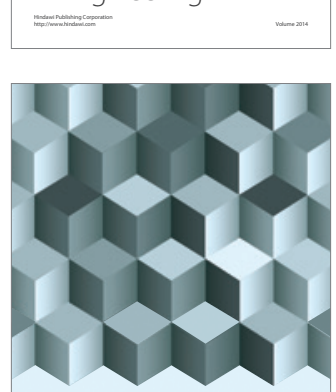

Journal of

Function Spaces
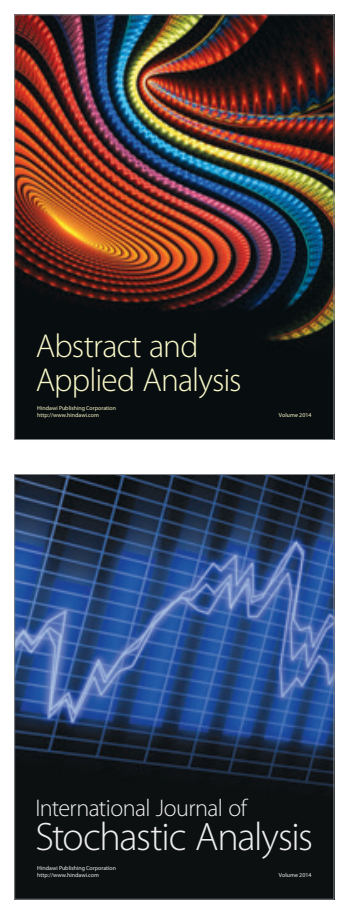

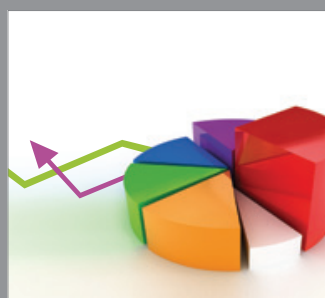

ournal of

Probability and Statistics

Promensencen
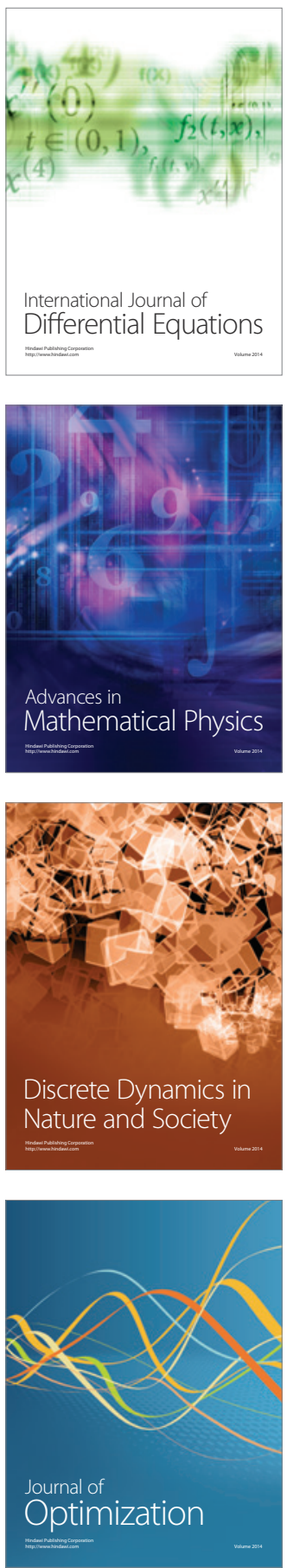